\title{
The rise of robots in surgical environments during COVID-19
}

\author{
Ajmal Zemmar ${ }^{1,2,3 凶}{ }^{\text {Andres M. Lozano }}{ }^{3}$ and Bradley J. Nelson ${ }^{4}$
}

\begin{abstract}
The COVID-19 pandemic has changed our world and impacted multiple layers of our society. All frontline workers and in particular those in direct contact with patients have been exposed to major risk. To mitigate pathogen spread and protect healthcare workers and patients, medical services have been largely restricted, including cancellation of elective surgeries, which has posed a substantial burden for patients and immense economic loss for various hospitals. The integration of a robot as a shielding layer, physically separating the healthcare worker and patient, is a powerful tool to combat the omnipresent fear of pathogen contamination and maintain surgical volumes. In this Perspective, we outline detailed scenarios in the pre-, intra- and postoperative care, in which the use of robots and artificial intelligence can mitigate infectious contamination and aid patient management in the surgical environment during times of immense patient influx. We also discuss cost-effectiveness and benefits of surgical robotic systems beyond their use in pandemics. The current pandemic creates unprecedented demands for hospitals. Digitization and machine intelligence are gaining significance in healthcare to combat the virus. Their legacy may well outlast the pandemic and revolutionize surgical performance and management.
\end{abstract}

T he original intention of robotic surgery was to permit the conduction of a surgical procedure from a remote distance without touching the patient ${ }^{1}$. The first surgical robots were the Arthrobot, which followed voice commands to assist in patient positioning during an orthopaedic surgical case ${ }^{2}$, and the Unimation Puma 200, used to orient a needle for brain biopsy3. In 1996, the Automated Endoscopic System for Optimal Positioning (AESOP), the first FDA-approved robotic surgical system, introduced ZEUS, a complete robotic surgical system with seven degrees of freedom, tremor elimination and motion scaling ${ }^{4}$. ZEUS was used for the first long-distance telesurgical procedure, a laparoscopic cholecystectomy on a French patient in Strasbourg, while the surgeon was located in New York ${ }^{5}$. Another breakthrough was the Da Vinci robotic system by Intuitive Surgery, which is used across different surgical specialties for a variety of surgical procedures and is capable of performing technically challenging procedures ${ }^{6,7}$. Several other robotic systems have now been developed and are commercially available for various surgical procedures across different disciplines (for a review see ref. ${ }^{7}$ ).

\section{Robotic technology in the age of pandemics}

Robots have long served humans to protect them from hazardous tasks. The first industrially successful robot, the Unimate, began operation at a General Motors assembly plant in New Jersey, performing automated tasks dangerous to humans ${ }^{4}$. Other examples are mobile robots aiding firefighters in environments with restricted visibility ${ }^{8}$, robots mimicking hand and arm movements conducted by a human at a safe distance to reduce health risks for vulnerable workers, for example, in nuclear environments ${ }^{9}$, and robotic aid in mountain rescue ${ }^{10}$. The current COVID-19 pandemic brings along several threats and restrictions to our society. A variety of potential implementations have been proposed to utilize robots in healthcare and beyond to face these challenges ${ }^{11,12}$. Healthcare workers are at the battle's forefront during pandemics and a professional group with high vulnerability. During the 2003 outbreak of severe acute respiratory syndrome (SARS) in Canada, $51 \%$ of the 438 cases were healthcare workers including three fatal cases $^{13}$. One of the earliest reports from the epicentre in Wuhan (China) found 40 healthcare workers among the first 138 patients $^{14}$, with infection rates up to $20 \%{ }^{15}$. As of July 2020 , more than 1,800 identified healthcare workers from 64 countries had died of COVID-19, the youngest being 20 and the eldest $99^{16}$. An updated list from the end of August 2020 counted 1,079 fatal cases of healthcare workers in the United States ${ }^{17}$.

Even among the best equipped centres and most developed nations, a shortage of personal protective equipment (PPE) is a key concern $^{15}$, which can create fierce competition between governments and prioritize medical staff in some countries over others. Another limiting factor is labour. While machines in healthcare systems could potentially operate beyond maximum capacity over longer periods and ventilators or wards can be urgently manufactured, healthcare workers cannot follow this pace ${ }^{15}$, creating a limiting factor in patient management and care during high-demand periods. The use of robots can aid in both of these examples, (1) reducing contact between the patient and healthcare provider and thus the need for PPE and (2) serving at maximum capacity during extraordinary times.

\section{Robotic technology in the surgical environment}

Surgical care is a foundation of any healthcare system with both elective and emergency operations contributing to the health services of the population. Operating rooms can be high-risk areas for transmission given the urgency in management, involvement of multiple teams, and high-risk activities. As a result of the substantial threat to surgeons during COVID-19, several surgical societies have published safety guidelines during procedures ${ }^{18,19}$ (see Supplementary Information). To effectively reduce pathogen spread, robots can

'Department of Neurosurgery, Henan Provincial People's Hospital, Henan University People's Hospital, Henan University School of Medicine, Zhengzhou, China. ${ }^{2}$ Department of Neurosurgery, School of Medicine, University of Louisville, Louisville, KY, USA. ${ }^{3}$ Department of Neurosurgery, Toronto Western Hospital, University of Toronto, Toronto, Ontario, Canada. ${ }^{4}$ Multi-Scale Robotics Laboratory, Swiss Federal Institute of Technology (ETH) Zurich, Zurich, Switzerland.凶e-mail: ajmal.zemmar@ulp.org 
be integrated at several sections of the sequence, which each surgical patient traverses during a hospital stay. This workflow can be divided into pre-operative care, anaesthesia, the surgical procedure and postoperative care, and the overall goal of robotic integration is to minimize contact between the patient and healthcare provider at each step.

Pre- and postoperative care. The triage process is often the first step after entering an emergency department. This process begins with information exchange between the paramedic team or patient and the triage staff. Logistical and medical information can be unified into a coordinating system calculating the routes to the hospital and considering the patient influx at nearby hospitals to make the journey to the hospital and triage process more efficient and decrease wait times ${ }^{20-22}$. Robotic technology and artificial intelligence (AI) can further be utilized to greet patients during pandemics, disinfect hands and distribute $\mathrm{PPE}^{23}$. Primary transmission of the severe acute respiratory syndrome coronavirus 2 (SARS-CoV-2) is through respiratory droplets and contact routes ${ }^{24}$ as well as faecal transmission ${ }^{25}$. Sterilization, defined as the process of killing microorganisms, is integral for avoiding pathogen contamination in the surgical environment. The sterility-assurance level (SAL) is specified as the probability of an item being non-sterile after undergoing sterilization. Implantable medical devices, for example, achieve an SAL of $10^{-6}$, meaning they have a probability of finding one viable microorganism in one million sterilized items ${ }^{26}$. A critical measure to contain pathogens is frequent cleaning. SARS-CoV-2 can persist up to 72 hours on surfaces ${ }^{27}$. Continuous robotic, non-contact ultraviolet surface disinfection can efficiently reduce contamination and has been used in hospitals ${ }^{28,29}$. This autonomous paradigm is employed at international airports to reduce human exposure to hazardous microorganisms and compensate for a shortage of labour. Robotic systems providing real-time feedback of contamination levels in contained rooms represent another tool for providing a safe working environment ${ }^{30}$. Using $\mathrm{AI}$, the Canadian company BlueDot was the first to predict the virus outbreak in Wuhan. Robots paired with AI could determine areas of high movement in the operating environment or the entire hospital and focus on more frequent sterilization of high-traffic areas. Besides droplet and contact, blood transmission is another route for pathogen spread. Each year, approximately 600,000-800,000 needle stick injuries occur in the United States, translating to up to US\$2,000 per day. This results in more than US\$0.5-1 billion of annual healthcare $\operatorname{cost}^{31}$. Portable robotic devices are able to use deep learning for autonomous blood vessel access despite anatomic variability, and can provide superior success rates in shorter time compared to human-employed manual access ${ }^{32}$. Especially in times of high demand, these technologies can mitigate contamination risk to healthcare workers and reduce economic burden. A critical and repetitive component of pre- and postoperative care is measurement of vital parameters, which in times of pandemics enables, for example, identification and separation of patients with fever. Robotic measurement of vital signs and bedside nursing assistance can not only reduce infectious spread ${ }^{33}$ but also reduce cost and effectiveness of care ${ }^{34,35}$. Besides a patient's vital signs, robots can deliver food or drugs on predefined criteria to reduce nurse-to-patient interaction. The use of intelligent robots for measurement of vital signs and drug delivery is currently being tested at Harvard's medical hospitals to mitigate disease transmission. Another example is the use of robots for autonomous transportation of drugs in a hospital in Guangzhou (China) during the COVID-19 outbreak. China's Wuhan Wuchang Hospital demonstrated during COVID-19 that these ideas can be implemented. At this hospital a smart field was created, in which 14 robots performed a number of tasks to take care of patients including cleaning, disinfection, drug delivery and temperature measurement ${ }^{36,37}$.
Anaesthesia. The anaesthetic team is one of the most vulnerable due to direct exposure and close contact to the patient's airway. Robotic airway management and intubation has been demonstrated ${ }^{38,39}$ and is a critical component for protecting healthcare workers during this potentially dangerous task, especially for infectious spread by airborne transmission. Other examples include semi-automated or automated anaesthesia, where the depth of anaesthesia is measured, and anaesthetics are delivered automatically. The same principle can be applied to maintain blood pressure, muscle relaxation and the level of anaesthetics during procedures performed under general anaesthesia or awake surgery.

Surgical procedure. The first identified COVID-positive patient in the United States was held in an isolated room designed during the Ebola crisis ${ }^{40}$. A robot equipped with a camera, a microphone and a stethoscope allowed patient consulting without physical contact between the patient and the doctor. Although the patient did not undergo an operation, today's technology potentially allows surgeries to occur remotely without need for the surgeon to enter the room. Besides distancing the surgeon, today's robotic systems typically require less staff in the operating room than with traditional open surgery (Fig. 1), which reduces the personnel exposed to infectious aerosols, further reducing the risk of contamination. Fully contactless surgical suites must be considered in the light of the current pandemic.

Two main factors dictate surgical guidelines during the current COVID-19 pandemic. First, stress on the healthcare system and, in particular, on intensive care unit (ICU) beds and equipment such as respirators and surgical facilities, as these resources are limited in a hospital that may face a sudden patient influx resulting in cancellation of elective procedures and reorganization of ICU beds. Second, the risk of infectious spread among patients and healthcare providers. Surgical robots can effectively face both of these challenges to perform time-efficient surgery over extensive operative hours and minimize infectious spread, for example, through contactless surgery, which is defined as no physical contact between the patient and the healthcare provider during the operation. These principles could be applied to a variety of disciplines (for a detailed review see ref. ${ }^{7}$ ). Major surgical societies have addressed specific guidelines during COVID-19 (see Supplementary Information), of which some have argued against the use of minimal-invasive procedures. To investigate this concern, two recently published articles compared the advantages and disadvantages of robot-assisted surgery (RAS) against conventional laparoscopy and their relevance during the COVID-19 pandemic ${ }^{41,42}$. Laparoscopic surgeries require establishment of an artificial pneumoperitoneum, which is generated by abdominal insufflation with $\mathrm{CO}_{2}$, helium, nitrous oxide or oxygen. While traditional laparoscopy requires pressure levels of 10-15 $\mathrm{mm} \mathrm{Hg}$, RAS can operate at physiologic intra-abdominal pressure levels up to $5 \mathrm{~mm} \mathrm{Hg}\left(\right.$ ref. ${ }^{43}$ ). Increased intra-abdominal pressure generates aerosols that can increase the risk for pathogen contamination to the surgical team ${ }^{41}$, that is, higher pressure levels entail increased aerosol generation, which brings along increased infectious risk. These cases argue that RAS can minimize contamination risk through reduced aerosol generation compared to traditional laparoscopic surgery. Another example is pathogen spread through smoke generated by surgical instruments. Ultrasonic devices and other equipment used during laparoscopic surgery can generate substantial amounts of smoke, which can contain pathogens such as Corynebacterium, papillomavirus and human immunodeficiency virus (HIV) and contaminate doctors through this route $e^{44-47}$. Strikingly, it was reported that particle concentration on a surgical knife was ten times higher in traditional laparoscopic surgery compared to non-laparoscopic cases $^{48}$. Low gas mobility in the established pneumoperitoneum is a potential explanation for the increased pathogen concentration ${ }^{41}$, which is likely to be lower in 
a

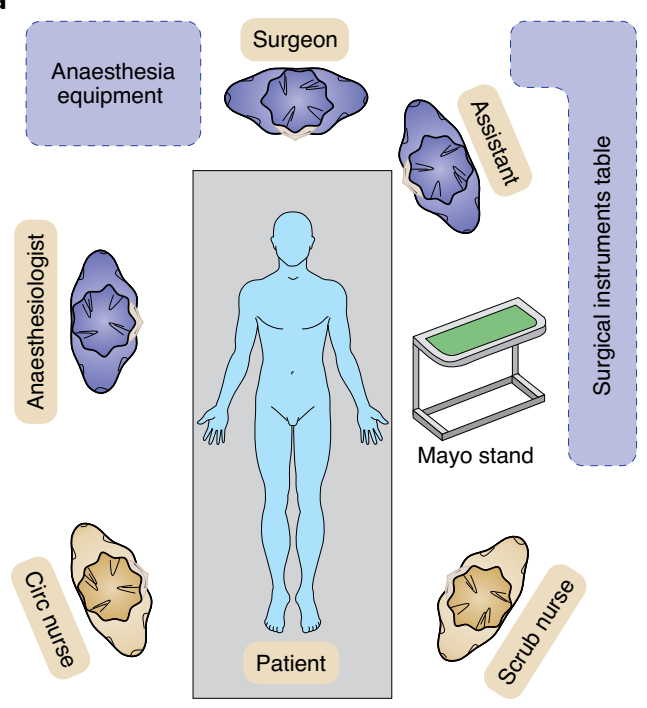

b

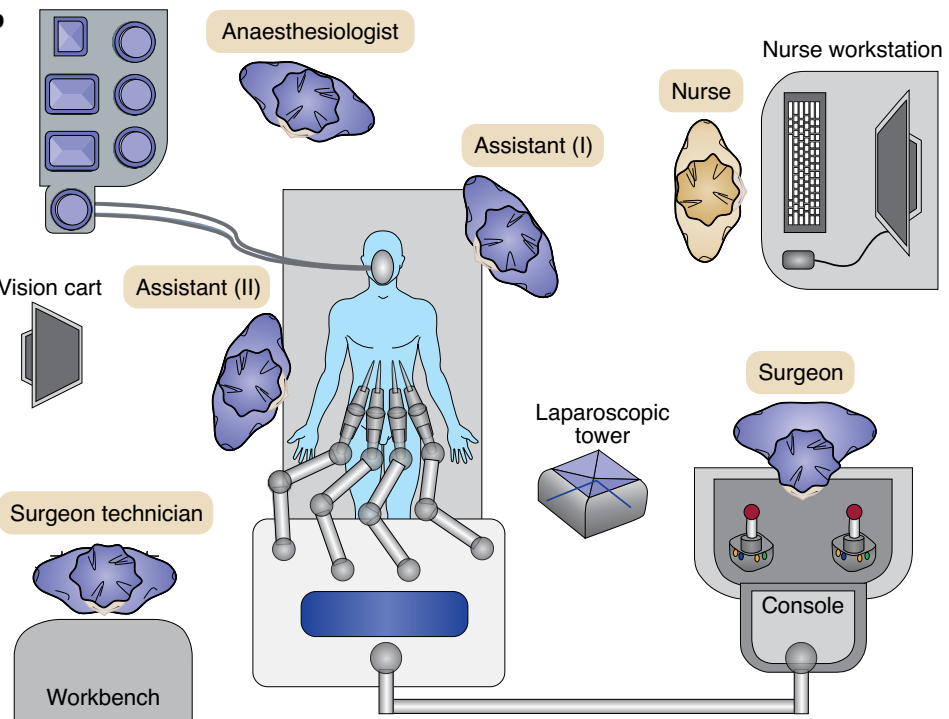

C

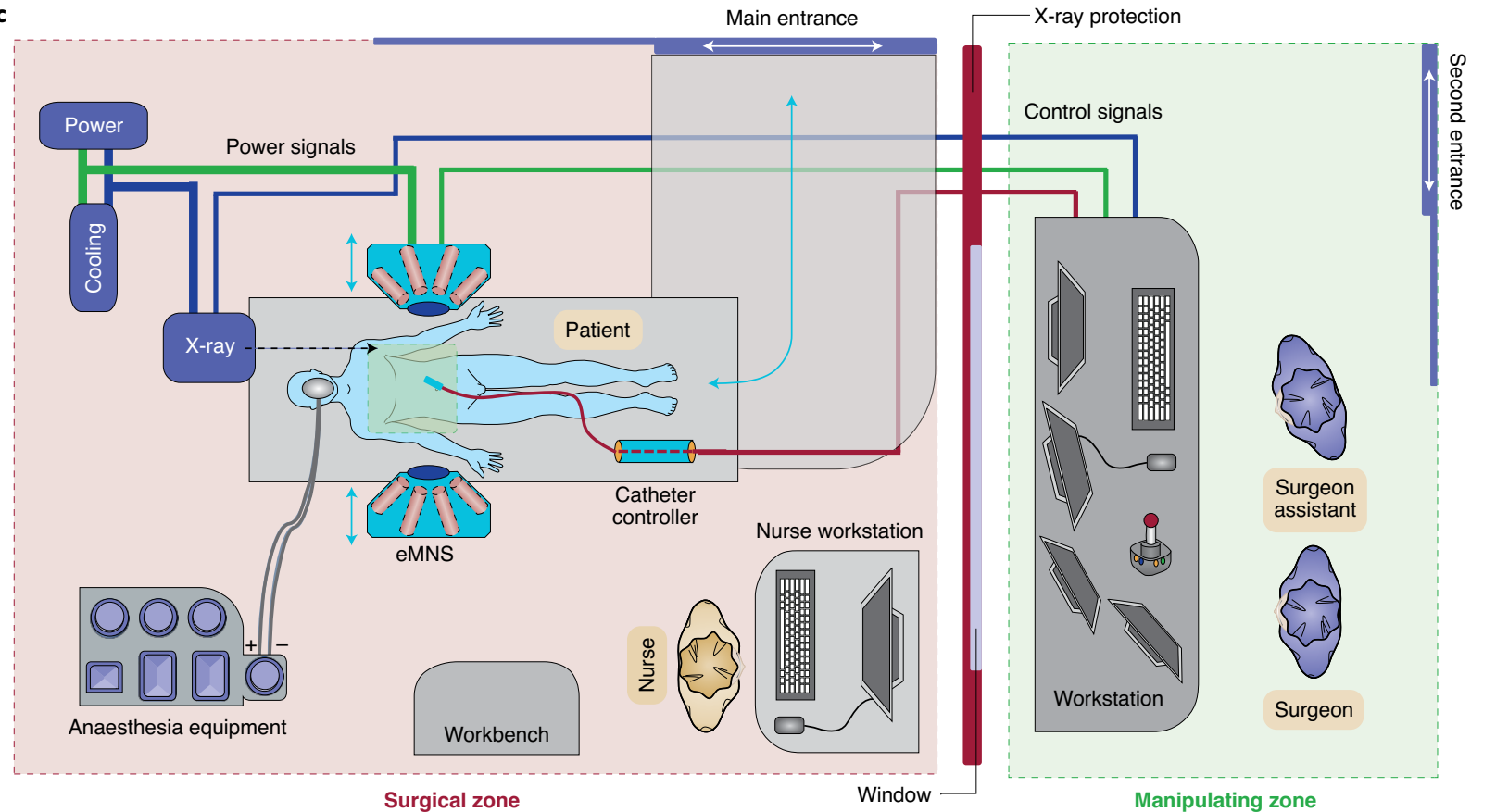

Fig. 1 | Operative room setup. a, Traditional operative room setup. b, Current operative room setup during a robotic surgery procedure. The surgeon directs the robot remotely to carry out the operation. Compared to $\mathbf{a}$, a reduced number of team members is in direct contact with the patient. $\mathbf{c}$, State of the art in contactless robotic surgery, where the entire team operates from a safe distance to the patient. An ablation procedure is performed on an isolated patient. The surgeon sits in a control zone outside the operating theatre and directs the surgery from a remotely controlled electromagnetic navigation system (eMNS).

RAS procedures, resulting in decreased infection risk. In addition to these observations, fewer surgical instruments are needed with RAS compared to the classic surgical approach, resulting in reduced risk for contamination and faster cleaning. Another important aspect is reduced duration of hospital stay with robotic surgery, which is especially relevant in times of shortage of hospital beds and potential of contamination with longer hospital admission.

Contactless robotic surgery with remote supervision sparing the presence of healthcare providers in the surgical room represents the ultimate scenario to reduce pathogen spread (Fig. 1c). An emerging technology towards this goal is magnetic navigation systems (MNS). Magnets placed external to the patient's body are used to guide a surgical probe equipped with a magnetic tip. While earlier systems were large, difficult to integrate to the clinical routine workflow and accompanied undesired movement of the needle tip after turning off the magnetic field ${ }^{49-52}$, more recent systems have overcome these challenges ${ }^{53,54}$. Using this technology, contactless surgery has been demonstrated in endovascular cases (Fig. 2c-e), enabling surgery at times when patient-to-healthcare provider contact is a substantial concern. In addition to pathogen mitigation, radiation exposure to healthcare workers is reduced as they operate outside the surgical suite. Paired with innovative catheters allowing independent control of stiffness and direction at separate segments (Fig. 2e) to further maximize the degree of steering freedom ${ }^{55}$, MNS 
a

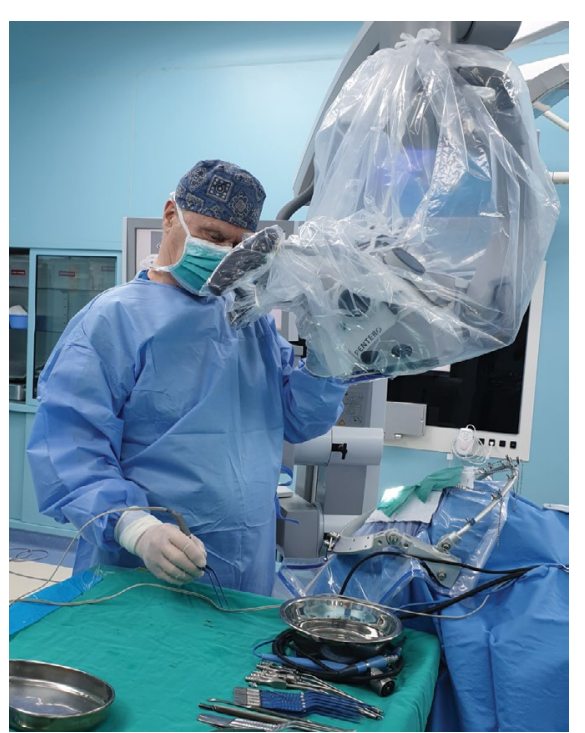

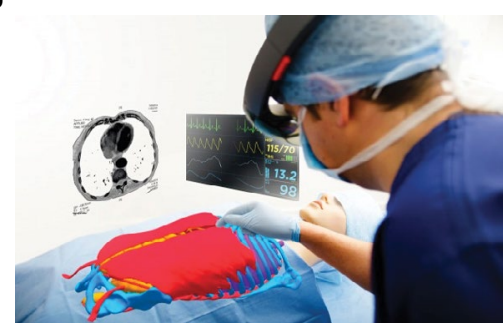

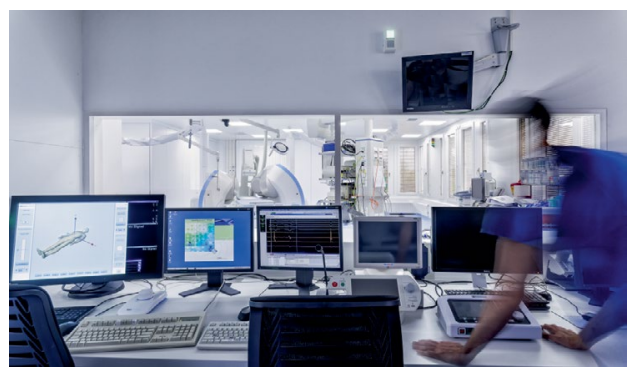

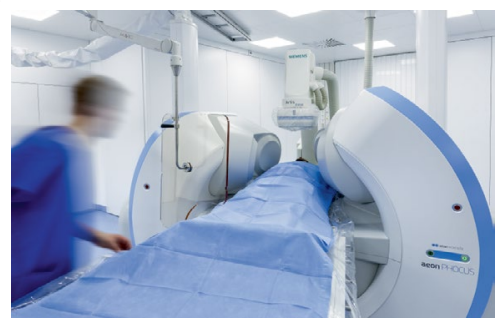

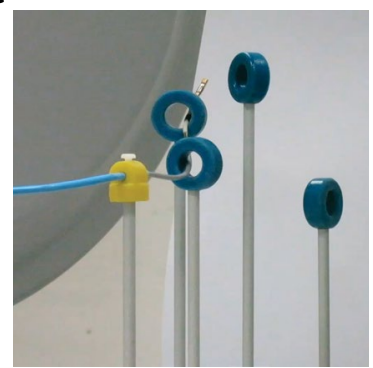

Fig. 2 | Centralization of intraoperative information to the surgeon. a, Current procedure during a neurosurgical case. The surgeon views the operative field through a microscope. Note the bipolar held in the right hand. The surgeon moves their eyes away from the microscopic view to search for the bipolar pedal to activate it. b, Digital surgical glasses unifying relevant information to the surgeon such as critical structures in the operative field, relevant imaging, vital parameters and so on. c,d, Contactless surgery procedure. The operative suite is shown in $\mathbf{c}$, and the surgeon's control zone is shown in $\mathbf{d}$. e, Example of an endovascular catheter steered with a magnetic navigation system. Credit: Juha Hernesniemi (a); Cambridge Consultants (b); Multi-Scale Robotics Lab (MSRL), ETH Zurich (c-e)

provide superb dexterity control of the probe resulting in increased procedural safety. Future applications using untethered microrobots controlled with magnetic steering ${ }^{56}$ can address targeted drug delivery or prevent viral adherence in high-conglomerate infectious areas of the body.

\section{Economical and psychological aspects of elective surgery cancellations}

During the COVID-19 pandemic, elective operations have been stopped in hospitals across a large number of countries. This measure entails (1) a tremendous economic impact for hospitals and individuals, and (2) substantial psychological consequences for affected patients. Elective surgeries represent a major income source for hospitals ${ }^{57}$. Without this source of revenue, hospitals are forced to reduce their employees' salaries, halt new appointments, and in a worst-case, declare bankruptcy. North America's largest hospital, New York Presbyterian Hospital, had budgeted a US\$246 million income for 2020; with the COVID-19 crisis it expects the operating losses alone to sum to between US\$104 million and US $\$ 454$ million $^{58}$. Smaller hospitals that are not located in majorly affected areas not only lose income from cancelled elective cases and outpatient clinics, but also experience a decrease in the volume of emergency room visits as people stay home. As a result, hospitals may experience low capacities over several weeks during declared shutdowns.

From the patient's perspective, cancellation of non-emergent surgery can have compelling psychological consequences. Among others, the Ontario Ministry of Health in Canada has compared wait times from June 2019 to June 2020 and found a substantial increase in wait times across different surgical subspecialties (Table 1). Patients scheduled for elective operations may suffer a substantial decrease in quality of life, for example, by living with a pain- or anxiety-triggering condition ${ }^{59}$. Paired with isolation during home stay and the public's concerns during a pandemic, these fears can have substantial mental health consequences. The use of robotic technology not only aids to reduce infectious spread during
Table 1 | Surgical wait times in the province of Ontario, Canada in days across different surgical specialties in June 2019 and June 2020

\begin{tabular}{lll} 
Surgical service area & June 2019 & June 2020 \\
\hline General surgery & 107 & 157 \\
Gynaecologic surgery & 136 & 190 \\
Neurosurgery & 158 & 203 \\
Oncology surgery & 50 & 90 \\
Opthalmic surgery & 208 & 245 \\
Oral and maxillofacial surgery and dentistry & 190 & 176 \\
Orthopaedic surgery & 213 & 246 \\
Otolaryngic surgery & 196 & 251 \\
Paediatric surgery & 167 & 209 \\
Plastic and reconstructive surgery & 146 & 208 \\
Thoracic surgery & 88 & 181 \\
Urologic surgery & 94 & 130 \\
Vascular surgery & 76 & 113 \\
\hline Sorce: Onaro Ho
\end{tabular}

Source: Ontario Health - Wait Time Information System.

pandemics and permit continuation of elective surgeries, but also provides care for isolated patients through social robots ${ }^{60}$. Furthermore, surgical robots can also be used to clear surgical backlog as they can perform surgery at weekends or after hours under supervision, helping with surgeon fatigue and error and permitting operation with reduced staff when compared to traditional surgery. To get more accurate information, modelling studies and eventually trials are needed to compare and predict infectious spread during a pandemic with and without the use of robotic technology in a hospital setting. 


\section{Economic considerations and benefits of surgical robots beyond pandemics}

Hospital administrations weigh the cost and benefit when deciding to purchase new equipment. The benefit of robots during pandemics has been outlined above, but is it worth acquiring surgical robotic systems just to survive a pandemic? Will surgical robots have usefulness to justify the investment beyond the end date of a pandemic? Implementation of robotic systems in the surgical environment can harbour clinical benefits by assisting the surgeon to improve surgical safety and to make the procedure more efficient resulting in reduced time and cost when running operative rooms. Here, we outline specific aspects in which robots improve the surgical workflow and procedure, potentially resulting in clinical and economic gain.

Machine learning and data analysis. Experience is a vital part to master surgery. Surgeons often analyse videos of their own procedures to learn from their previous cases to improve for the next case. Future systems permit automated data recording and analysis. Using deep learning and AI, these systems open new possibilities for surgical applications, leading to reduced complications and development of autonomous robotic systems for an increased range of procedures. These technologies also open new avenues for training and evaluating surgeons ${ }^{61,62}$ and, vice versa, open a new field to train surgeons on how to operate robots ${ }^{63}$.

Centralizing vital information to the surgeon. In aviation or automobiles, the pilot or the driver accesses a central console to which all relevant information is projected. Currently, the surgeon receives information about vital parameters from the anaesthesiologist, accesses imaging on separate devices to locate critical structures, and translates the information seen in imaging into the surgical field. These steps dramatically interrupt the surgical flow. For each of these tasks, the surgeon has to look away from the operative field, which causes increased operative time and cost. Developing systems to centralize the needed information will substantially lessen this burden. Today's technologies remain mostly separated depending on the focus of the respective manufacturer. Uniting the microscope view, imaging used for intra-operative navigation and virtual and augmented reality into one centralized viewing cockpit for the surgeon (for example, smart surgical glasses; Fig. 2) allows unprecedented visualization of critical structures projected directly into the surgeon's operative field. Haptic feedback paired to the surgical instrument for tactile feedback when approaching critical structures and online information of vital parameters further centralize the information displayed to the surgeon in one central console ${ }^{64,65}$.

Enhancing the senses to gain intraoperative flexibility. Although voice-driven devices allow us to communicate with machines in daily life and despite efforts made by the very first robotic system and ZEUS's AESOP system, this technology has not yet routinely found its way into the operating theatre. For example, the surgeon has to move his eyes away from the operative field to find the pedal for the bipolar coagulation device, then adapt his eyes back to the surgical field and press the pedal to activate coagulation (Fig. 2a). As bipolar cautery is used frequently during surgery, this repeated sequence of actions substantially prolongs the entire procedure. To avoid this interruption, some surgeons have incorporated the scrub nurse to press the bipolar pedal after a voice command, which allows them to keep their eyes on the surgical field. A simple voice-driven bipolar instrument could effectively aid to improve this repetitive task, make one of the most frequently used intraoperative steps more efficient and spare repetitive action of the scrub nurse. Eye tracking is another powerful approach to let the surgeon communicate verbally with perioperative technologies ${ }^{66}$.
Telesurgery, software development and 5G connectivity. In addition to allowing the surgeon to operate from a safe distance during wars or pandemics, remote surgery has several other important implications. In large territories such as Canadian and Chinese provinces, or the American states, patients are currently flown from distant areas with restricted medical centres to hospital centres in large cities, which comes with substantial cost for the flight crew, medical staff and other components of the transportation chain. Telesurgical systems have been implemented to overcome this hurdle. However, telesurgery included lags in connection speed. Recently introduced 5G connectivity can overcome signal delay and permits signal transmission in real time. As there are generally more general surgeons than sub-specialized surgeons in most locations across the globe, telesurgical procedures could be conducted by a general surgeon on site and supervised by a sub-specialized surgeon remotely who can interact in the operative field through a virtual interface. Other examples for this paradigm include remote surgery conducted by trained specialists in underdeveloped parts of the globe or for medical tourism. This approach decreases the need for travel and reduces cost as well as the carbon dioxide footprint. While implementing these novel technologies, ethical concerns must be faced in detail, especially in the highly sensitive field of healthcare, which large technology companies have aimed to enter for a long time.

Surgical effectiveness and reduced operative time: minimal to not measurable clinical benefits have been observed when early robotic systems were compared with traditional surgery ${ }^{7}$. However, this is changing rapidly as more advanced systems are being developed and as surgeons become more familiar and increasingly efficient with their use ${ }^{67-69}$. Shorter hospital stays and faster recovery observed with robotic surgery can further contribute to cost reduction and improve clinical outcomes as desired in paradigms such as enhanced recovery after surgery ${ }^{70}$. A vital aspect in adopting robotic surgery is the surgeon's acceptance of innovative technology. Seamless integration into clinical routines by avoiding major adaptations for the surgeon's and the operative team's workflow is an important aspect in adopting the new technology. Examples of this include the newer generations of Intuitive Surgical's Da Vinci system, which allows efficient mounting to the operative table, and the CardioArm and Flex robotic systems ${ }^{71,72}$, which are mounted directly to the patient's body or can even be handheld to simplify their integration and ease of use for the surgeon. To create efficient designs, effective communication between the clinical team, the engineering team and the commercial vendor is essential, as early on in project conception as possible. Such collaborative approach among clinicians, engineers and robotics companies have rendered more user-friendly robotic systems in the recent years, which have been increasingly implemented in clinical use. Continuation of this development is likely to result in shorter procedure times and overall cost saving, which plays an important role in cost-calculation in purchasing robotic systems ${ }^{73}$. In addition, increased competition on the market will render lower purchasing prices in the near future ${ }^{67}$.

\section{Conclusion}

The current pandemic reveals how much our activities are reliant on human-to-human physical contact. In surgical environments, this necessity poses a substantial challenge during COVID-19. To avoid pathogen spread, elective surgeries are cancelled with considerable physical, mental and economic consequences for patients and hospitals. AI and robotic technology can be utilized to face this challenge. Outside the operating room, these technologies can be implemented for various tasks such as digitized patient admission, effective triaging during times of high demand, acquisition and monitoring of vital signs, identification of high-risk nodes, sterilization with real-time contamination feedback, drawing of blood and delivery of food and drugs. In the operating theatre, robots can place 
intravascular lines, intubate the patient and manage the airway. For the surgical procedure itself, the smaller the number of the surgical team, the less the risk of contamination. While robot-assisted surgery can reduce contamination risk, contactless remote robotic surgery would be the ideal scenario to prevent pathogen spread as it technically can be conducted with only the presence of the patient in the operating room. This visionary setting would allow continuing surgery during pandemics without risking increased contamination. Modelling studies based on accurate contact tracing in the operating room to compare detailed scenarios in the surgical environment with and without the use of robots would help to estimate the true benefit of robots in containing pathogen spread.

Beyond the pandemic, use of these technologies in surgical environments can provide other benefits to improve safety and efficiency for the patient and to serve rural areas more effectively through remote surgery. Utilization of AI, virtual and augmented reality can help the surgeon to make the procedure safer, for example, by haptic feedback and projection of critical structures within the surgical field. Centralizing the assistive tools provided to the surgeon into a single component, for example, into a surgical glass, can simplify the application to the surgeon.

History shows that technology has advanced surgery more than any other field. Examples include the invention of imaging, the cautery, the microscope and many more. AI, machine learning and robotic technology may well be the next quantum leap.

Received: 2 June 2020; Accepted: 13 September 2020;

Published online: 13 October 2020

\section{References}

1. Satava, R. M. Surgical robotics: the early chronicles: a personal historical perspective. Surg. Laparosc. Endosc. Percutaneous Tech. 12, 6-16 (2002).

2. Smith, J. A., Jivraj, J., Wong, R. \& Yang, V. 30 years of neurosurgical robots: review and trends for manipulators and associated navigational systems. Ann. Biomed. Eng. 44, 836-846 (2016).

3. Kwoh, Y. S., Hou, J., Jonckheere, E. A. \& Hayati, S. A robot with improved absolute positioning accuracy for CT guided stereotactic brain surgery. IEEE Trans. Biomed. Eng. 35, 153-160 (1988).

4. Ranev, D. \& Teixeira, J. History of computer-assisted surgery. Surg. Clin. North Am. 100, 209-218 (2020).

5. Marescaux, J. \& Rubino, F. in Teleophthalmology (eds Yogesan, K. et al.) 261-265 (Springer, 2006)

6. Marescaux, J. et al. Transatlantic robot-assisted telesurgery. Nature 413, 379-380 (2001).

7. Troccaz, J., Dagnino, G. \& Yang, G.-Z. Frontiers of medical robotics: from concept to systems to clinical translation. Annu. Rev. Biomed. Eng. 21 193-218 (2019).

8. Xing, Y. et al. Mobile robot multi-sensor unit for unsupervised gas discrimination in uncontrolled environments. In Proc. IEEE Sensors (IEEE, 2017)

9. Tuffield, P. \& Elias, H. The shadow robot mimics human actions. Ind. Robot 30, 56-60 (2003)

10. Sugiyama, H., Tsujioka, T. \& Murata, M. Real-time exploration of a multirobot rescue system in disaster areas. Adv. Robot. 27, 1313-1323 (2013)

11. Yang, G. Z. et al. Combating COVID-19-the role of robotics in managing public health and infectious diseases. Sci. Robot. 5, eabb5589 (2020).

12. Tavakoli, M., Carriere, J. \& Torabi, A. Robotics, smart wearable technologies, and autonomous intelligent systems for healthcare during the COVID-19 pandemic: an analysis of the state of the art and future vision. Adv. Intell. Syst. 2, 2000071 (2020).

13. Moore, D., Gamage, B., Bryce, E., Copes, R. \& Yassi, A. Protecting health care workers from SARS and other respiratory pathogens: organizational and individual factors that affect adherence to infection control guidelines. Am. J. Infect. Control. 33, 88-96 (2005).

14. Wang, D. et al. Clinical characteristics of 138 hospitalized patients with 2019 novel coronavirus-infected pneumonia in Wuhan, China. J. Am. Med. Assoc. 323, 1061-1069 (2020).

15. COVID-19: protecting health-care workers. Lancet 395, 922 (2020).

16. In memoriam: healthcare workers who have died of COVID-19. Medscape https://www.medscape.com/viewarticle/927976 (2020).

17. Lost on the frontline: hundreds of US healthcare workers have died fighting Covid-19. We count them and investigate why. The Guardian https://www. theguardian.com/us-news/ng-interactive/2020/aug/11/lost-on-the-frontlinecovid-19-coronavirus-us-healthcare-workers-deaths-database (2020).
18. Givi, B. et al. Safety recommendations for evaluation and surgery of the head and neck during the COVID-19 pandemic. JAMA Otolaryngol. Head Neck Surg. 146, 579-584 (2020).

19. Chiu, P. W. Y., Hassan, C., Yip, H. C., Antonelli, G. \& Sharma, P. ISDE guidance statement management of upper-GI endoscopy and surgery in COVID-19 outbreak. Diseases Esophag. 33, doaa029 (2020).

20. Wilkes, D. M. et al. Heterogeneous artificial agents for triage nurse assistance. In 10th IEEE-RAS Int. Conf. Humanoid Robots (IEEE, 2010).

21. Chang, C. \& Murphy, R. R. Towards robot-assisted mass-casualty triage. In IEEE Int. Conf. Networking, Sensing and Control (IEEE, 2007).

22. Burke, R. V. et al. Using robotic telecommunications to triage pediatric disaster victims. J. Pediatr. Surg. 47, 221-224 (2012).

23. Robots to greet, triage incoming coronavirus patients. Medgadget https://www.medgadget.com/2020/03/robots-to-greet-triage-incomingcoronavirus-patients.html (2020).

24. Li, Q. et al. Early transmission dynamics in Wuhan, China, of novel coronavirus-infected pneumonia. N. Engl. J. Med. 382, 1199-1207 (2020).

25. Hindson, J. COVID-19: faecal-oral transmission? Nat. Rev. Gastroenterol. Hepatol. 17, 259 (2020).

26. Baird, R. M. in Russell, Hugo and Ayliffe's Principles and Practice of Disinfection, Preservation and Sterilization 4th edn (eds Fraise, A. P. et al.) 787-799 (2008).

27. van Doremalen, N. et al. Aerosol and surface stability of SARS-CoV-2 as compared with SARS-CoV-1. N. Engl. J. Med. 382, 1564-1567 (2020).

28. Kovach, C. R. et al. Evaluation of an ultraviolet room disinfection protocol to decrease nursing home microbial burden, infection and hospitalization rates. BMC Infect. Dis. 17, 186 (2017).

29. Fleming, M. et al. Deployment of a touchless ultraviolet light robot for terminal room disinfection: the importance of audit and feedback. Am. J. Infect. Control 46, 241-243 (2018).

30. Kraft, K., Chu. T., Hansen, P. \& Smart, W. D. Real-time contamination modeling for robotic health care support. In IEEE Int. Conf. Intelligent Robots and Systems (IEEE, 2016).

31. Cooke, C. E. \& Stephens, J. M. Clinical, economic, and humanistic burden of needlestick injuries in healthcare workers. Med. Devices Evid. Res. 10, 225-235 (2017).

32. Chen, A. I., Balter, M. L., Maguire, T. J. \& Yarmush, M. L. Deep learning robotic guidance for autonomous vascular access. Nat. Mach. Intell. 2, 104-115 (2020).

33. Machiel Van der Loos, H. F., Ullrich, N. \& Kobayashi, H. Development of sensate and robotic bed technologies for vital signs monitoring and sleep quality improvement. Auton. Robots 15, 67-79 (2003).

34. Broadbent, E. et al. The cost-effectiveness of a robot measuring vital signs in a rural medical practice. In Proc. IEEE Int. Workshop on Robot and Human Interactive Communication (IEEE, 2015).

35. Lee, H., Piao, M., Lee, J., Byun, A. \& Kim, J. The purpose of bedside robots: exploring the needs of inpatients and healthcare professionals. Comput. Inform. Nurs. 38, 8-17 (2020).

36. Wittbold, K. A., Carroll, C., Iansiti, M., Zhang, H. M. \& Landman, A. B. How hospitals are using AI to battle Covid-19. Harvard Business Review https:// hbr.org/2020/04/how-hospitals-are-using-ai-to-battle-covid-19 (2020).

37. Kent, C. How are robots contributing to the fight against coronavirus? Medical Device Network https://www.medicaldevice-network.com/features/ coronavirus-robotics/ (2020).

38. Boehler, Q. et al. REALITI: a robotic endoscope automated via laryngeal imaging for tracheal intubation. IEEE Trans. Med. Robot. Bionics 2, 157-164 (2020)

39. Biro, P. et al. Automated tracheal intubation in an airway manikin using a robotic endoscope: a proof of concept study. Anaesthesia 75, 848-851 (2020).

40. Holshue, M. L. et al. First case of 2019 novel coronavirus in the United States. N. Engl. J. Med. 382, 929-936 (2020).

41. Zheng, M. H., Boni, L. \& Fingerhut, A. Minimally invasive surgery and the novel coronavirus outbreak: lessons learned in China and Italy. Ann. Surg. 272, e5-e6 (2020).

42. Kimmig, R., Verheijen, R. H. M. \& Rudnicki, M. Robot assisted surgery during the COVID-19 pandemic, especially for gynecological cancer: a statement of the Society of European Robotic Gynaecological Surgery (SERGS). J. Gynecol. Oncol. 31, e59 (2020).

43. Angioli, R. et al. Influence of pneumoperitoneum pressure on surgical field during robotic and laparoscopic surgery: a comparative study. Arch. Gynecol. Obstet. 291, 865-888 (2015).

44. Capizzi, P. J., Clay, R. P. \& Battey, M. J. Microbiologic activity in laser resurfacing plume and debris. Lasers Surg. Med. 23, 172-174 (1998).

45. Hensman, C., Baty, D., Willis, R. G. \& Cuschieri, A. Chemical composition of smoke produced by high-frequency electrosurgery in a closed gaseous environment: an in vitro study. Surg. Endosc. 12, 1017-1019 (1998).

46. Johnson, G. K. \& Robinson, W. S. Human immunodeficiency virus-1 (HIV-1) in the vapors of surgical power instruments. J. Med. Virol. 33, 47-50 (1991). 
47. Gloster, H. M. \& Roenigk, R. K. Risk of acquiring human papillomavirus from the plume produced by the carbon dioxide laser in the treatment of warts. J. Am. Acad. Dermatol. 32, 436-441 (1995).

48. Li, C. I., Pai, J. Y. \& Chen, C. H. Characterization of smoke generated during the use of surgical knife in laparotomy surgeries. J. Air Waste Manag. Assoc. 70, 324-332 (2020).

49. Yuan, S. et al. Long-term outcomes of the current remote magnetic catheter navigation technique for ablation of atrial fibrillation. Scand. Cardiovasc. J. 51, 308-315 (2017).

50. McNeil, R. G. et al. Functional design features and initial performance characteristics of a magnetic-implant guidance system for stereotactic neurosurgery. IEEE Trans. Biomed. Eng. 42, 793-801 (1995).

51. Grady, M. S. et al. Magnetic stereotaxis: a technique to deliver stereotactic hyperthermia. Neurosurgery 27, 1010-1016 (1990).

52. Quate, E. G. \& Gillies, G. T. Nonlinear magnetic stereotaxis: three-dimensional, in vivo remote magnetic manipulation of a small object in canine brain. Med. Phys. 17, 405-415 (1990).

53. Petruska, A. J. et al. Magnetic needle guidance for neurosurgery: initial design and proof of concept. In Proc. IEEE Int. Conf. Robotics and Automation (IEEE, 2016).

54. Hong, A. et al. 3D path planning for flexible needle steering in neurosurgery. Int. J. Med. Robot. Comput. Assist. Surg. 15, e1998 (2019).

55. Chautems, C., Tonazzini, A., Floreano, D. \& Nelson, B. J. A variable stiffness catheter controlled with an external magnetic field. In IEEE Int. Conf. Intelligent Robots and Systems (IEEE, 2017).

56. Nelson, B. J., Kaliakatsos, I. K. \& Abbott, J. J. Microrobots for minimally invasive medicine. Annu. Rev. Biomed. Eng. 12, 55-85 (2010).

57. Herrod, P. J. J. et al. Winter cancellations of elective surgical procedures in the UK: a questionnaire survey of patients on the economic and psychological impact. BMJ Open 9, e028753 (2019).

58. Singh, S. \& Coleman-Lochner, L. Hospitals burn through cash while congress weighs next stimulus. Bloomberg https://www.bloomberg.com/news/ articles/2020-03-31/hospitals-burn-through-cash-while-congress-weighsnext-stimulus (2020)

59. Carr, T., Teucher, U., Mann, J. \& Casson, A. G. Waiting for surgery from the patient perspective. Psychol. Res. Behav. Manag. 2, 107-119 (2009).

60. Aymerich-Franch, L. Why it is time to stop ostracizing social robots. Nat. Mach. Intell. 2, 364 (2020).

61. Dunkin, B., Adrales, G. L., Apelgren, K. \& Mellinger, J. D. Surgical simulation: a current review. Surg. Endosc. Other Interv. Tech. 21, 357-366 (2007).

62. Evans, C. H. \& Schenarts, K. D. Evolving educational techniques in surgical training. Surg. Clin. North Am. 96, 71-88 (2016).

63. Sridhar, A. N., Briggs, T. P., Kelly, J. D. \& Nathan, S. Training in robotic surgery-an overview. Curr. Urol. Rep. 18, 58 (2017).

64. Visarius, H., Gong, J., Scheer, C., Haralamb, S. \& Nolte, L. P. Man-machine interfaces in computer assisted surgery. Comput. Aided Surg. 2, 102-107 (1997).
65. O'Hara, K. et al. Touchless interaction in surgery. Commun. ACM 57, 70-77 (2014).

66. Stephan, D., Sälzer, H. \& Willeke, F. First experiences with the New Senhance telerobotic system in visceral surgery. Visc. Med. 34, 31-36 (2018).

67. Casarin, J. et al. Implementing robotic surgery for uterine cancer in the United States: better outcomes without increased costs. Gynecol. Oncol. 156, 451-458 (2020)

68. Agarwal, R., Rajanbabu, A., Goel, G. \& Unnikrishnan, U. G. A comparison of the clinical outcomes in uterine cancer surgery after the introduction of robotic-assisted surgery. J. Obstet. Gynecol. India 69, 284-291 (2019)

69. Yun, J. E. et al. Clinical outcomes and costs of robotic surgery in prostate cancer: a multiinstitutional study in Korea. Prostate Int. 7, 19-24 (2019).

70. Ljungqvist, O., Scott, M. \& Fearon, K. C. Enhanced recovery after surgery: a review. JAMA Surg. 152, 292-298 (2017).

71. Payne, C. J., Rafii-Tari, H. \& Yang, G. Z. A force feedback system for endovascular catheterisation. In IEEE Int. Conf. Intelligent Robots and Systems (IEEE, 2012).

72. Payne, C. J. \& Yang, G. Z. Hand-held medical robots. Ann. Biomed. Eng. 42, 1594-1605 (2014).

73. Feldstein, J., Schwander, B., Roberts, M. \& Coussons, H. Cost of ownership assessment for a da Vinci robot based on US real-world data. Int. J. Med. Robot. Comput. Assist. Surg. 15, e2023 (2019).

\section{Acknowledgements}

This work was supported by grants from the Heidi Demetriades Foundation, the ETH Zurich Foundation, and the Henan Provincial People's Hospital Outstanding Talents Founding Grant Project to A.Z. We would like to thank J. Hernesniemi, Z. Chen, Z. Sheng, G. Hongri and E. Zuurmond for fruitful discussions and assistance with the images in Fig. 2.

\section{Competing interests}

A.M.L. has a consulting role for Medtronic, Abbott, Boston Scientific and Functional Neuromodulation.

\section{Additional information}

Supplementary information is available for this paper at https://doi.org/10.1038/ s42256-020-00238-2.

Correspondence should be addressed to A.Z .

Reprints and permissions information is available at www.nature.com/reprints.

Publisher's note Springer Nature remains neutral with regard to jurisdictional claims in published maps and institutional affiliations.

(C) Springer Nature Limited 2020 